\title{
MEK inhibition exhibits efficacy in human and mouse neurofibromatosis tumors
}

\author{
Walter J. Jessen, ${ }^{1}$ Shyra J. Miller, ${ }^{1}$ Edwin Jousma, ${ }^{1}$ Jianqiang Wu, ${ }^{1}$ Tilat A. Rizvi, ${ }^{1}$ \\ Meghan E. Brundage, ${ }^{1}$ David Eaves, ${ }^{1}$ Brigitte Widemann, ${ }^{2} \mathrm{Mi}-\mathrm{OK}$ Kim, ${ }^{3}$ Eva Dombi, ${ }^{2}$ \\ Jessica Sabo, ${ }^{2}$ Atira Hardiman Dudley, ${ }^{1}$ Michiko Niwa-Kawakita, ${ }^{4}$ Grier P. Page, ${ }^{5}$ \\ Marco Giovannini, ${ }^{6}$ Bruce J. Aronow, ${ }^{7}$ Timothy P. Cripe, ${ }^{1}$ and Nancy Ratner ${ }^{1}$
}

\begin{abstract}
${ }^{1}$ Division of Experimental Hematology and Cancer Biology, Children's Hospital Medical Center, Cincinnati, Ohio, USA. ${ }^{2}$ Pediatric Oncology Branch, National Cancer Institute, Bethesda, Maryland, USA. ${ }^{3}$ Biostatistics and Epidemiology, Department of Pediatrics,

Cincinnati Children's Hospital Medical Center, Cincinnati, Ohio, USA. ${ }^{4}$ Institut National de la Santé et de la Recherche Médicale, Paris, France. ${ }^{5}$ Statistics and Epidemiology Unit, RTI International, South Chamblee, Georgia, USA. ${ }^{6}$ Department of Neural Tumor Research, House Ear Institute, Los Angeles, California, USA. Biomedical Informatics, Cincinnati Children's Hospital Medical Center, Cincinnati, Ohio, USA.
\end{abstract}

\begin{abstract}
Neurofibromatosis type 1 (NF1) patients develop benign neurofibromas and malignant peripheral nerve sheath tumors (MPNST). These incurable peripheral nerve tumors result from loss of NF1 tumor suppressor gene function, causing hyperactive Ras signaling. Activated Ras controls numerous downstream effectors, but specific pathways mediating the effects of hyperactive Ras in NF1 tumors are unknown. We performed crossspecies transcriptome analyses of mouse and human neurofibromas and MPNSTs and identified global negative feedback of genes that regulate Ras/Raf/MEK/ERK signaling in both species. Nonetheless, ERK activation was sustained in mouse and human neurofibromas and MPNST. We used a highly selective pharmacological inhibitor of MEK, PD0325901, to test whether sustained Ras/Raf/MEK/ERK signaling contributes to neurofibroma growth in a neurofibromatosis mouse model $\left(N f^{f l / f l} ; D h b\right.$-Cre $)$ or in NF1 patient MPNST cell xenografts. PD0325901 treatment reduced aberrantly proliferating cells in neurofibroma and MPNST, prolonged survival of mice implanted with human MPNST cells, and shrank neurofibromas in more than $80 \%$ of mice tested. Our data demonstrate that deregulated Ras/ERK signaling is critical for the growth of NF1 peripheral nerve tumors and provide a strong rationale for testing MEK inhibitors in NF1 clinical trials.
\end{abstract}

\section{Introduction}

The hallmark manifestation of neurofibromatosis type 1 (NF1) is the development of peripheral nerve tumors (1). In benign and malignant peripheral nerve tumors, cells acquire somatic inactivation of the second NF1 allele and loss of NF1 (neurofibromin) protein function (2). With few exceptions, NF1 patients develop small benign dermal neurofibromas that can number into the thousands and be extremely disfiguring. At least a third of NF1 patients develop larger benign plexiform neurofibromas that cause disfigurement and morbidity when they compress vital structures. Surgical removal of neurofibromas is not always feasible due to tumor location, resulting in substantial morbidity for NF1 patients, and plexiform neurofibromas can transform to malignant peripheral nerve sheath tumors (MPNSTs), a leading cause of death in adults with NF1 (3-6). There is currently no chemotherapeutic regimen that will effectively treat NF1 tumors, warranting investigation into the development of novel molecular-targeted therapeutic strategies (7). Studies have begun to identify molecular alterations in MPNST tumors, yet the pathway of molecular events contributing to neurofibroma growth or progression to malignancy remains unclear (8).

Neurofibromin is a Ras-GTPase activating protein (Ras-GAP), converting active Ras-GTP to inactive Ras-GDP $(9,10)$. Therefore, cells isolated from neurofibromas (11) and MPNSTs $(12,13)$ that lack neurofibromin have elevated levels of active Ras-GTP. Aberrant activation of Ras signaling generally leads to promotion of tumor cell proliferation and/or survival (14). However, in benign

Conflict of interest: The authors have declared that no conflict of interest exists. Citation for this article: J Clin Invest. 2013;123(1):340-347. doi:10.1172/JCI60578. tumors, Ras signaling can alternatively be associated with oncogene-induced senescence $(15,16)$, and evidence supports a role for cellular senescence in NF1 dermal neurofibromas (17).

At least 11 effector pathways downstream of Ras-GTP have been described (18). Research focused on the biology of NF1 and pathogenesis of plexiform neurofibroma and MPNST has identified potential therapeutic targets including Ras itself, Ras effectors, growth factor receptors, and angiogenesis $(8,19)$. For example, Ral and PI3K/AKT/mTOR/S6K1, where mTOR indicates mammalian target of rapamycin, are known to regulate cell proliferation, survival, and cell death and have each been implicated in NF1 tumorigenesis $(20,21)$. S6K1 is activated in MPNST cells with NF1 mutations, and this response is attenuated by rapamycin in MPNST cell lines, MPNST xenografts, and in a genetic engineered mouse model with $N f 1$ and $p 53$ mutations in cis $(22,23)$. On this basis, a phase II trial of rapamycin in plexiform neurofibromas is ongoing. However, no chemotherapeutic approach blocking any molecular target, including tyrosine kinases upstream of Ras, Ras, Ras effectors, or combination of effectors, has to date prevented or arrested neurofibroma formation or more than transiently delayed MPNST growth (7).

Genetically engineered mouse (GEM) Nf1 models have been developed using Cre/lox technology for ablation of Nf1 (24-28). We chose the $N f 1^{f l / f l} ; D h b$-Cre model for preclinical testing, as the neurofibroma histology in this model replicates human neurofibroma histology $(25,26)$. While some mouse models of neurofibroma formation, and perhaps some human patients, require a heterozygous genetic background (29), the $N f 1^{f l / f l}$;Dhb-Cre model does not, facilitating preclinical testing. We have used 7 Tesla small-animal MRI to assess tumor growth rate in the $\mathrm{Nf1} 1^{f l f l}$; Dhb-Cre 


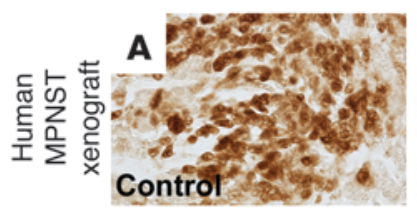

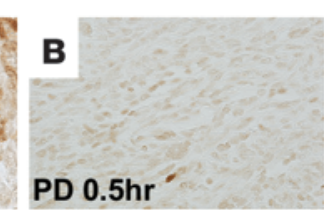

$\mathbf{F}$

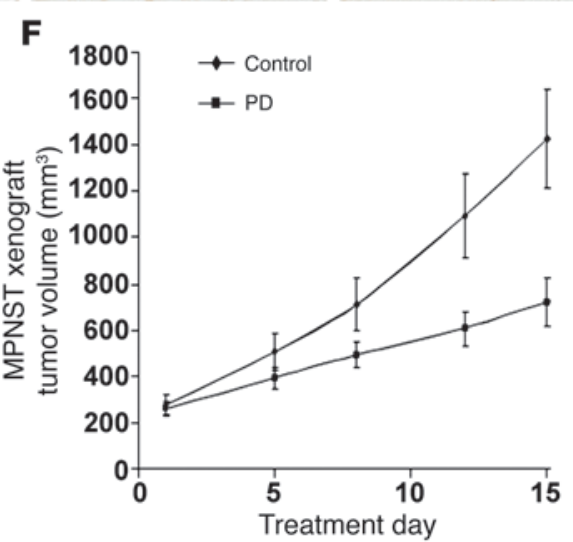

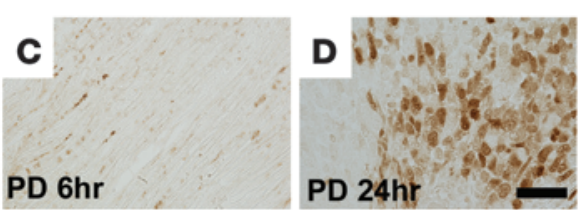

G
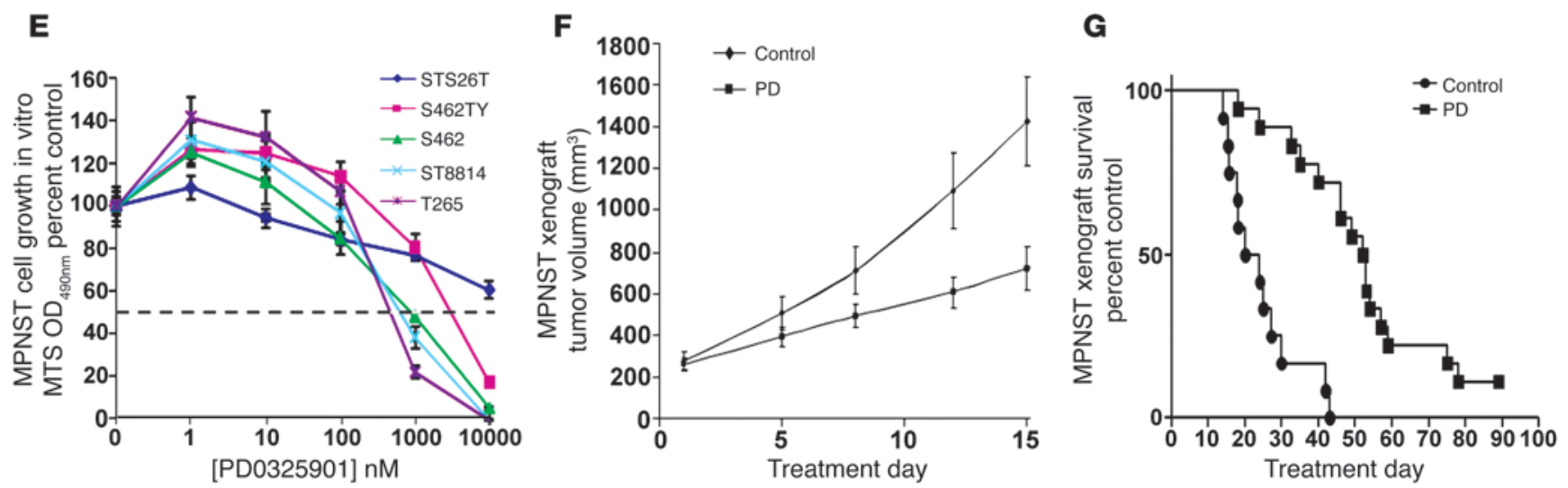

Figure 1

PD0325901 reduces p-ERK and inhibits MPNST cell growth. (A-D) Brown staining indicates detection of p-ERK in paraffin tissue sections. p-ERK is robust in human S462TY MPNST xenografts (A), but is absent 30 minutes after treatment with $10 \mathrm{mg} / \mathrm{kg}$ PD0325901 (PD) (B). p-ERK remains detectable at low levels at 6 hours after treatment (C) and returns to pretreatment levels by 24 hours (D). Scale bar: $50 \mu \mathrm{m}$. (E) Dose-response analysis of PD0325901 on 5 MPNST cell lines. Effect on cell growth is expressed as percentage of control, and PD0325901 concentration shows nM concentrations on a log scale. $(\mathbf{F})$ Tumor volume $\left(\mathrm{mm}^{3}\right)$ was significantly reduced in S462TY MPNST xenografts treated with PD0325901 $(n=18)$ versus control $(n=12)$. Reduction in tumor volume observed by treatment day 5 was maintained through day $15(P=0.004)$, when many control mice required sacrifice. (E and F) Error bars represent mean \pm SEM. (G) Survival of MPNST xenografted mice doubled with 3 months PD0325901 treatment $(P<0.0001)$.

mouse model using volumetric MRI analysis. However, treatment with the rapamycin analog RAD001 failed to block tumor growth, and the multikinase inhibitor sorafenib affected few mice (30). The same volumetric measurement technique is in use in ongoing clinical trials and has been proven to sensitively detect small changes in tumor size over time $(31,32)$. The reproducibility of this method is similar for tumors in mice and humans, and thus the response criteria used in human trials can be applied to the preclinical evaluation in mice. Here, we confirm the transcriptional similarities of human and mouse tumors using a bioinformatics approach and illustrate the use of our GEM model for preclinical evaluation of candidate molecular targets.

We compared the transcriptomes of human NF1 tumors and GEM Nf1 models to normal differentiated peripheral nerves of each species to identify molecular mechanisms contributing to tumorigenesis and shared potential therapeutic targets. Our results support the hypothesis that hyperactive Ras induces expression of genes that suppresses the canonical downstream pathway, Raf/MEK/ERK, in benign neurofibromas and MPNST. Although these transcriptional changes suggest that a negative feedback loop has been induced, ERK remains active in these tumors. Based on these data, we performed preclinical trials of the MEK inhibitor PD0325901 in mouse models of NF1-associated peripheral nerve tumors and observed remarkable efficacy. Similarly, elsewhere in this issue of the JCI, Chang and coworkers report dramatic responses to PD0325901 in a mouse model of juvenile myelomonocytic leukemia (JMML) characterized by homozygous $N f 1$ inactivation (33). Together, these data provide a strong rationale for targeting MEK in the treatment of NF1associated neoplasms.

\section{Results}

PD0325901 inbibits MEK and moderately reduces MPNST cell survival in vitro. To identify molecular similarities between mouse and human NF1 tumors, we performed cross-species transcriptome analysis of human NF1-derived peripheral nerve tumors, neurofibroma and MPNST $(n=32)(34)$, and mouse neurofibroma and MPNST $(n=33)$ compared with normal differentiated peripheral nerves of each species (Supplemental Table 1; supplemental material available online with this article; doi:10.1172/JCI60578DS1). Human data were publically available from our previous studies (34); mouse data have been deposited in the GEO database (GSE41747). Hierarchical clustering of orthologous transcripts defined expression clusters (C17-C20; Supplemental Figure 1A). To prioritize relevant genes, we queried transcripts from orthologous clusters (C17-C20; Supplemental Figure 1) representing significant transcriptional changes in mouse and human samples. Notably, both species upregulated expression of genes that negatively regulate the canonical Ras effector pathway Raf/MEK/ERK (Supplemental Table 2 and Supplemental Figure 1, B and C). Despite transcriptional upregulation of genes that repress Raf/MEK/ERK signaling, and consistent with neurofibroma (11) and MPNST $(12,13)$ cells having elevated levels of Ras-GTP, activation of the downstream effector ERK was maintained in neurofibromas and MPNSTs, as elevated levels of phosphorylated ERK (p-ERK) were detected in neurofibromas and MPNSTs (Supplemental Figure 2).

PD0325901 is a MEK inhibitor currently in clinical cancer trials $(35,36)$. Of 70 tested kinases, PD0325901 blocked MEK1 at values of $1 \mu \mathrm{M}$ in vitro, with the closely related MEK5 affected at 10 -fold higher concentrations (37); no other kinases were affected. The unusual specificity of this inhibitor is due to the fact that 

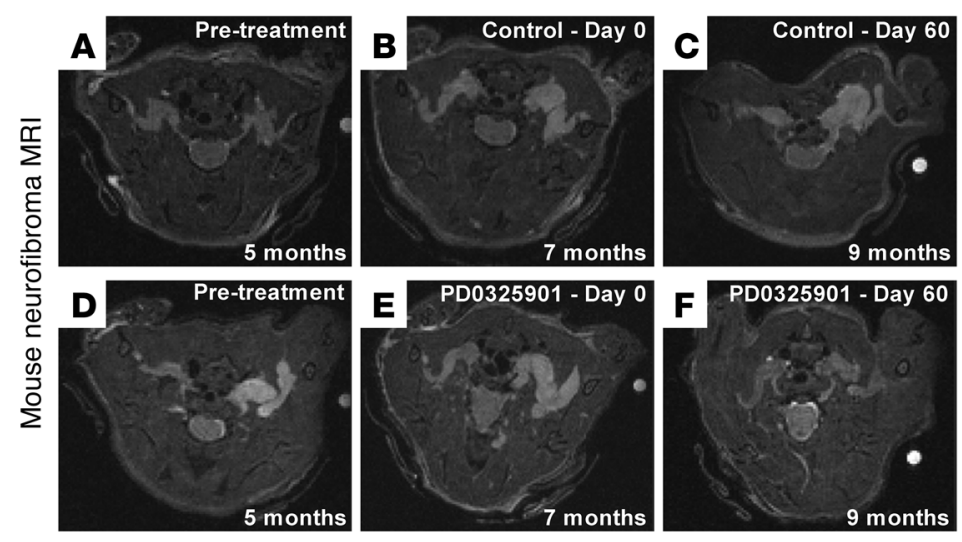

G

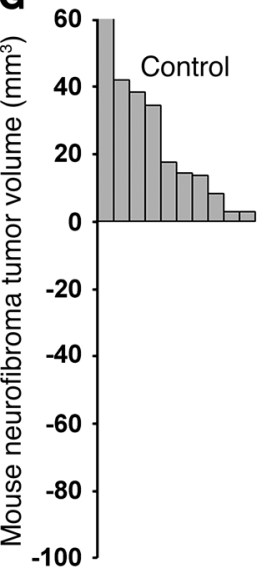

PD $1.5 \mathrm{mg} / \mathrm{kg}$

PD $5 \mathrm{mg} / \mathrm{kg}$

PD $10 \mathrm{mg} / \mathrm{kg}$

PD0325901 is not a direct kinase inhibitor, but rather an allosteric inhibitor. In comparison with its predecessor CI-1040, PD0325901 is more potent and has improved duration and bioavailability and increased metabolic stability (38). PD0325901 blocked Raf/MEK/ ERK signaling based on pharmacodynamic measurement of p-ERK in MPNST xenografts at intervals after PD0325901 exposure (Figure 1, A-D). p-ERK disappeared by 30 minutes after dosing MPNST xenografts (Figure 1B); levels remained low at 6 hours (Figure 1C) and resembled pretreatment levels by 24 hours (Figure 1D). Pharmacodynamic assessment of PD0325901 was at the end of the 60-day treatment period, demonstrating that the inhibitor remained efficacious throughout the experiment. Dose-response analysis of PD0325901 was conducted on 5 MPNST cell lines in vitro. Four MPNST cell lines were derived from NF1 patients (S462TY, S462, ST8814, T265); 1 MPNST cell line was derived from a sporadic MPNST (STS26T). After 4 days of treatment, effects on survival in the 4 NF1-derived cell lines were modest and variable ( $\mathrm{IC}_{50}$ values $420-3100 \mathrm{nM}$ (203-1495 ng/ml); (Figure 1E). Some of these concentrations were below the achievable trough plasma level $(250 \mathrm{ng} / \mathrm{ml})$ in humans (35); all were below the maximal documented achievable peak plasma level (1508 ng/ml) (36). Interestingly, the sporadic MPNST cell line (STS26T), expressing low levels of Ras-GTP (39), was least sensitive to PD0325901. Relative to other tumor cell lines, especially $B R A F$ mutant cells (average $\mathrm{IC}_{50}$ values $\left.<10 \mathrm{nM}\right)(40)$, MPNST cells were not particularly sensitive to MEK inhibition in vitro.

To exclude PD0325901 off-target effects, we utilized a MEK mutant, L115P, predicted to block interaction of MEK and PD0325901 (33). Expressing this mutant protein in the NF1-defi- cient tumor cell line MPNST 8814 blocked ERK activation and cell proliferation in PD0325901-treated cells. Importantly, expression of the mutant did not block the effects of PD098059, a structurally unrelated MEK inhibitor (Supplemental Figure 3).

To test the effect of MEK inhibition on MPNST in vivo, S462TY MPNST cells derived from an NF1 patient were implanted into $n u / n u$ mice (41) and treated with PD0325901 at a dose $(10 \mathrm{mg} / \mathrm{kg} / \mathrm{d})$ comparable to the dose previously tested in the clinic $(35,36,42)$. Inhibiting MEK activity diminished tumor growth (Figure 1F) and doubled survival (mean survival $=22.5 \mathrm{vs}$. 52.5 days; $P<0.0001$ ) (Figure $1 \mathrm{G}$ ). We did not observe complete suppression of tumor growth in vivo, supporting the moderate response to MEK inhibition in vitro.

Efficacy of PD0325901 on neurofibroma growth in vivo. In $\mathrm{Nf1}$ fl/fl; Dhb-Cre mice, the Nf1 gene is deleted exclusively in Schwann cells. All $N f 1^{f l / f l} ; D h b$-Cre mice develop multiple neurofibromas with histological identity to human neurofibromas (25). Nf1 $1^{f / f l} ; \mathrm{Dhb}$-Cre mice neurofibroma growth rates can be measured using serial volumetric MRI analysis (30). Neurofibroma growth rates vary among mice, mimicking human neurofibromas, which are also monitored by serial MRI. There is less than $10 \%$ variability in measurement of the same tumor from 2 individuals, and tumor size measured by MRI has been verified by direct dissection (43). We measured tumor volume at 5 (Figure 2, A and D) and 7 (Figure 2, $\mathrm{B}$ and $\mathrm{E})$ months. Eighteen tumor-bearing mice were randomly assigned to PD0325901 treatment $(10 \mathrm{mg} / \mathrm{kg} /$ day) (Figure $2 \mathrm{~F})$; 10 received vehicle (Figure $2 \mathrm{C}$ ). All tumors in an individual mouse responded equally to treatment. Therefore, we compared the sum of tumor volumes in each individual mouse before and after treat- 


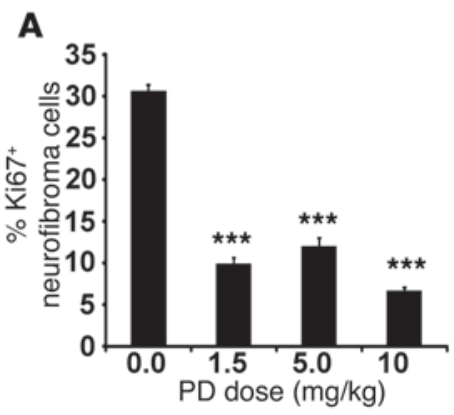

C
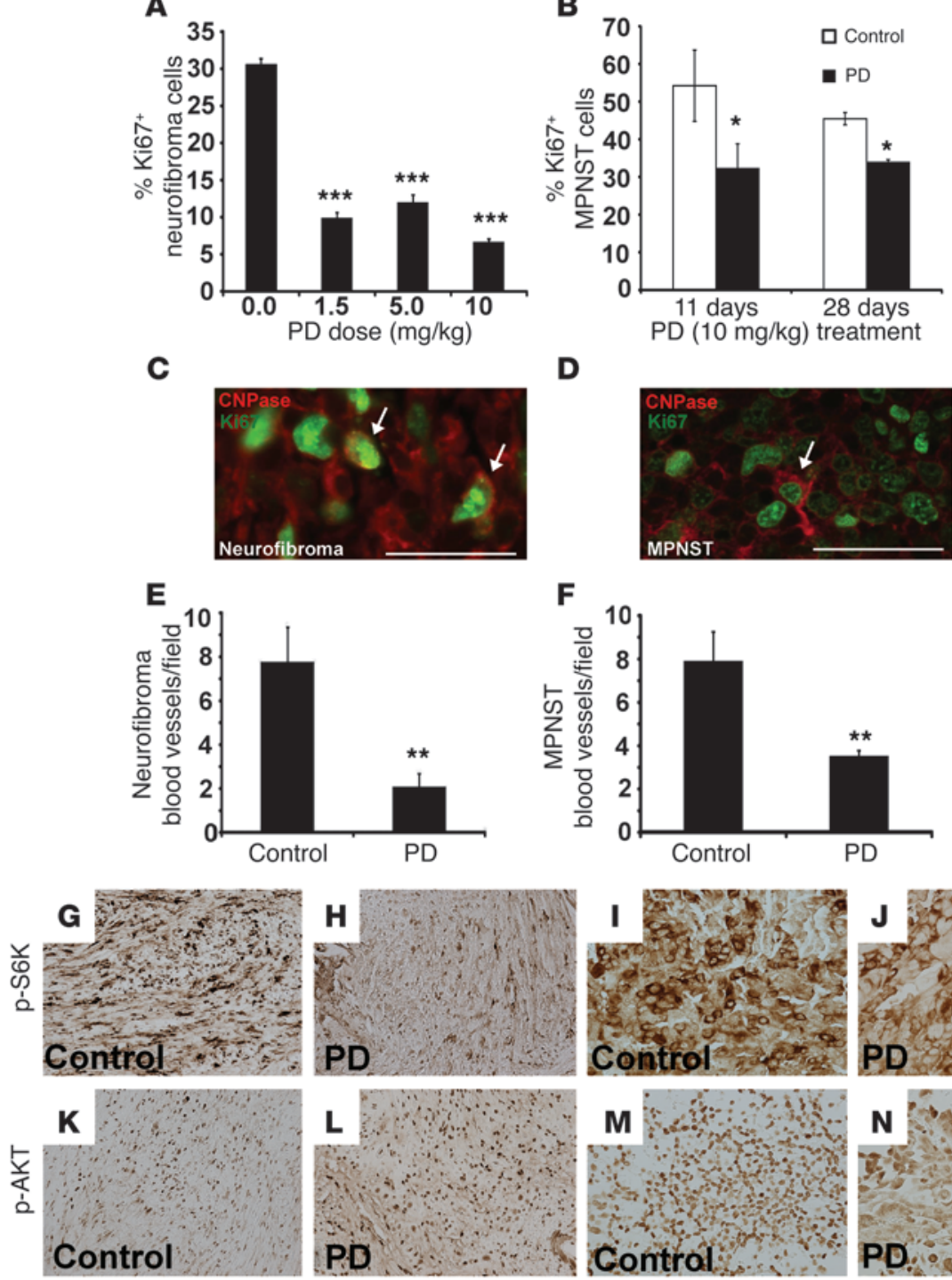

D
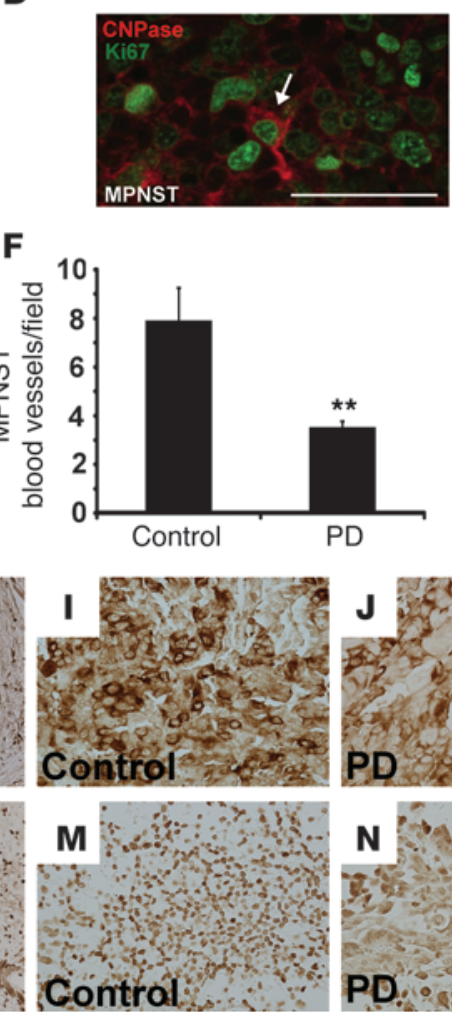

Figure 3

Molecular mechanism of PD0325901 in Nf1 ${ }^{f / f f} ;$; Dhh-Cre neurofibromas and MPNST xenografts. (A and B) Assessment of proliferation in neurofibromas and MPNSTs by quantification of Ki67 ${ }^{+}$cells as compared with hematoxylin-stained nuclei. (A) Neurofibromas in Nf $1^{f l f f l}$; Dhh-Cre mice treated with $1.5,5.0$, or $10 \mathrm{mg} / \mathrm{kg}$ PD0325901 for 60 days showed a significant $\left.{ }^{* * *} P<0.001\right)$ reduction in the percentage of Ki67+ cells relative to mice treated with control vehicle. (B) Mice harboring MPNST xenografts treated with PD0325901 for 11 days and 28 days show a significant $\left({ }^{*} P<0.05\right)$ reduction in the percentage of Ki67 ${ }^{+}$cells relative to mice treated with control vehicle. (C and D) Labeling of Ki67+ proliferating cells (green), CNPase ${ }^{+}$(red) Schwann cells, and DAPI+ (blue) cell nuclei in Nf1 fllfl; Dhh-Cre neurofibromas (C) and MPNST xenografts (D). Arrows represent double-label cells (Ki67+; CNPase ${ }^{+}$. Scale bar: $50 \mu \mathrm{m}$. (E and F) Assessment of vasculature in neurofibromas and MPNSTs by quantification of MECA+ endothelial cells. Number of blood vessels per high-powered field was significantly ( $\left.{ }^{\star \star} P<0.01\right)$ reduced in both neurofibromas $(\mathbf{E})$ and MPNSTs (F) in response to PD0325901. (G-J) p-S6K is detected in mouse neurofibromas $(\mathbf{G}$ and $\mathbf{H})$ and human MPNST xenografts (I and J); p-S6K levels decrease in response to treatment with PD0325901 $(\mathbf{H}$ and $\mathbf{J})$ relative to control $(\mathbf{G}$ and $\mathbf{I})$. (K-N) p-AKT is detected in mouse neurofibromas $(\mathbf{K}$ and $\mathbf{L})$ and human MPNST xenografts ( $\mathbf{M}$ and $\mathbf{N}$ ), and p-AKT levels do not change in response to treatment with PD0325901 ( $\mathbf{L}$ and $\mathbf{N}$ ) relative to control ( $\mathbf{K}$ and $\mathbf{M})$. Scale bars: $50 \mu \mathrm{m}$.

ment with vehicle or PD0325901. For statistical analyses, we also studied 20 historical controls, untreated mice, and vehicle-treated control mice from the same genotype and strain background imaged previously.
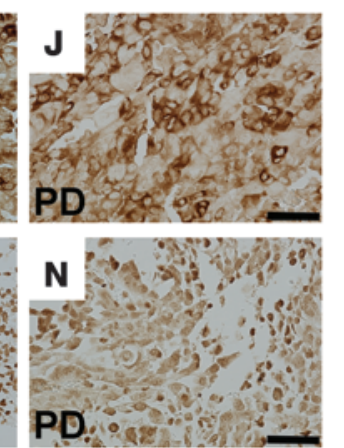

Because this dose of MEK inhibitor has been reported to cause toxicity in human trials after prolonged exposure (35, $36,42)$, we also analyzed mice for effects at lower doses of PD0325901. Sixteen mice were treated with $5 \mathrm{mg} / \mathrm{kg}$ and 15 mice with $1.5 \mathrm{mg} / \mathrm{kg}$ PD0325901. The $1.5 \mathrm{mg} / \mathrm{kg}$ PD0325901 dose in mice provided a concentration similar to that which will be tested in upcoming human trials ( $8 \mathrm{mg}$ BID). A striking reduction in neurofibroma volumes was achieved after 60 days of PD0325901 treatment (Figure 2G). Remarkably, tumor volumes were reduced in mice treated with $1.5 \mathrm{mg} / \mathrm{kg}(10 / 15$ mice), $5 \mathrm{mg} / \mathrm{kg}$ (15/16 mice), or $10 \mathrm{mg} / \mathrm{kg}$ (14/18 mice) PD0325901 $(P<0.001$ each dose; mixed models analysis; Supplemental Figure 4). PD0325901 was well tolerated with no apparent toxicity at any dose.

Reduced tumor volume suggested that cell proliferation or cell death might be altered in neurofibromas. Cell proliferation was decreased at the end point of PD0325901 treatment of $N f 1^{f l / f l} ; D b h$-Cre neurofibromas (Figure 3A). Similar effects on cell proliferation were observed at all 3 doses $(P<0.001)$. Apoptosis was not observed at the end of the experiment (not shown), but neurofibromas did regress. MPNST xenografts treated with PD0325901 showed a modest and marginally significant $(P<0.05)$ reduction in proliferating $\mathrm{Ki}^{+} 7^{+}$cells (Figure $3 \mathrm{~B}$ ) relative to neurofibromas (Figure 3A). Similar effects on MPNST proliferation were observed with short-term (11 days) or long-term (28 days) exposure to PD0325901 (Figure 3B). Double labeling tumor sections with $\mathrm{Ki} 67$ and CNPase, a Schwann cell marker, indicated that many of the proliferating cells were Schwann cells (Figure 3, C and D).

In addition to effects on tumor cell proliferation, the number of $\mathrm{MECA}^{+}$blood vessels was significantly reduced in both neurofibromas (Figure 3E; $P=0.003$ ) and MPNST (Figure 3F; $P=0.008$ ) subsequent to PD0325901 treatment. This may have resulted indi- 


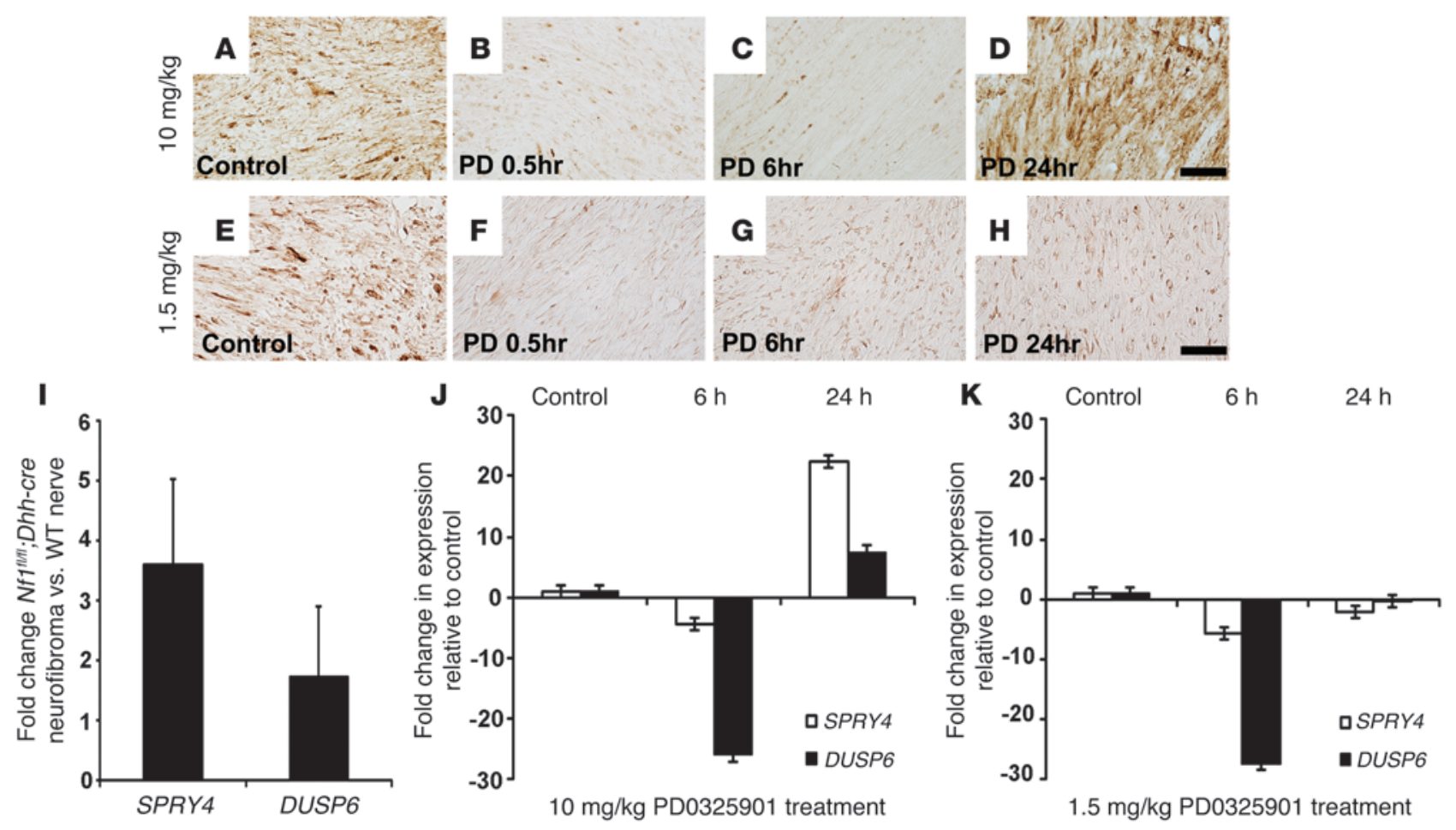

Figure 4

Negative feedback regulation of $p$-ERK in Dhh-Cre neurofibromas. (A-H) Brown staining indicates detection of $p$-ERK in paraffin tissue sections.

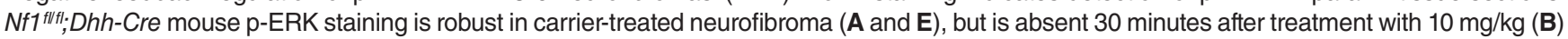
or $1.5 \mathrm{mg} / \mathrm{kg}$ (F) PD0325901. p-ERK becomes detectable 6 hours (C) after treatment with $10 \mathrm{mg} / \mathrm{kg}$ PD0325901 and returns to pretreatment levels by 24 hours (D). p-ERK also becomes detectable 6 hours (G) after treatment with $1.5 \mathrm{mg} / \mathrm{kg}$ PD0325901, but does not return to pretreatment levels by 24 hours (H). Scale bars: $50 \mu \mathrm{m}$. (I-K) qRT-PCR assessment of Ras pathway negative feedback. (I) Independent qRT-PCR confirmation of microarray data (Supplemental Figure 1B) showing overexpression of SPRY4 and DUSP6 in Nf1 ${ }^{f l f t} ;$ Dhh-Cre neurofibromas relative to WT mouse nerve. (J) Fold-change in SPRY4 and DUSP6 gene expression relative to pretreatment (control) at 6 and 24 hours after treatment with $10 \mathrm{mg} / \mathrm{kg}$ PD0325901, reflecting changes in p-ERK observed in (A-D). (K) Fold change in SPRY4 and DUSP6 gene expression relative to pretreatment (control) at 6 and 24 hours after treatment with $1.5 \mathrm{mg} / \mathrm{kg}$ PD0325901, reflecting changes in $\mathrm{p}$-ERK observed in $\mathrm{E}$ and $\mathbf{F}$. Error bars represent mean \pm SD.

rectly from effects of tumor cells on endothelial cells or directly through effects on blood vessels. In either case, changes in tumor vasculature likely contributed to changes in tumor volume.

Interestingly, we observed reduced p-S6K in PD0325901-treated $N f 1^{f l f l}$;Dhb-Cre neurofibromas, but not MPNSTs (Figure 3, G-J), suggesting that $\mathrm{S} 6 \mathrm{~K}$ is in part downstream of MEK in NF1 benign tumor cells. A reduction in p-S6K after MEK inhibition was also observed in melanoma cell lines (44). Ras/PI3K/Akt is a Ras pathway alternative to Ras/Raf/MEK/ERK, but PD0325901 did not affect p-AKT levels in neurofibromas or MPNSTs (Figure 3, K-N), validating PD0325901 specificity as a MEK inhibitor.

PD0325901 maintains MEK inhibition at low doses in Nf1 fl/fl; Dhb-Cre neurofibromas. To determine whether the Raf/MEK/ERK negative feedback mechanism suggested by the microarray data (Supplemental Figure 1, B and C) was valid, we analyzed p-ERK and expression of 2 candidate genes, SPRY4 and DUSP6, at the end of the 60-day treatment period of $N f 1^{f l f l}$; Dhb-Cre with low-dose $(1.5 \mathrm{mg} / \mathrm{kg})$ and high-dose $(10 \mathrm{mg} / \mathrm{kg})$ PD0325901. As observed with high-dose treatment of MPNST xenografts (Figure 1, A-D), p-ERK was reduced in $\mathrm{Nf} 1^{f l / f l}$; $D$ bh-Cre neurofibromas relative to control (Figure 4A) by 30 minutes (Figure 4B), remained low at 6 hours (Figure 4C), and was robust at 24 hours after the last dose of drug (Figure 4D). Reduced p-ERK was observed whether neurofibromas shrank or did not shrink after exposure to PD0325901 (not shown). Similar results were observed with the low-dose treatment at 30 minutes and 6 hours (Figure 4, E-G). However, in sections from mice treated with $1.5 \mathrm{mg} / \mathrm{kg}$ MEK inhibitor, p-ERK remained suppressed at 24 hours (Figure $4 \mathrm{H}$ ).

We confirmed overexpression of SPRY4 and DUSP6 in Nf1 $1^{f / f l}$; $D h b$-Cre neurofibromas relative to WT mouse nerve (Figure 4I). Expression of SPRY4 and DUSP6 mRNA was each reduced relative to vehicle control-treated mice at 6 hours following the last dose of PD0325901 at $10 \mathrm{mg} / \mathrm{kg}$ (Figure 4J) or $1.5 \mathrm{mg} / \mathrm{kg}$ (Figure 4K) PD0325901. However, a significant increase in expression at 24 hours was only observed with the higher dose (Figure 4, J and $\mathrm{K})$. These data suggest that different levels of MEK inhibition differentially affect accumulation of mRNAs encoding negative feedback regulators of ERK. Importantly, the data are consistent with the response of neurofibromas to single-agent MEK inhibition, even at low doses.

\section{Discussion}

A primary goal of this study was to identify and evaluate gene expression signatures shared between human NF1 tumors and GEM Nf1 models. We posited that shared signatures would reveal critical mechanisms of tumorigenesis and key therapeutic targets to facilitate preclinical studies and development of NF1 therapeutics. Functional enrichment analysis identified a prominent 
theme, Raf/MEK/ERK suppression, shared between mouse and human. Despite this transcriptional signature, ERK signaling remained activated in NF1 tumors and inhibiting Raf/MEK/ERK signaling with a MEK inhibitor diminished tumor cell growth. Our data set provides a wealth of gene expression information and supports MEK signaling as an important clinical target in NF1.

Our results identify a transcriptional response to suppress Raf/ MEK/ERK activity in neurofibromas and MPNST, including overexpression of DUSP and Sprouty family members, feedback inhibitors of Raf/MEK/ERK signaling. Expression of DUSP family members was reported in response to NF1 ablation in human fibroblasts (17) and KRAS2-activating mutations in lung tumors (45), suggesting a common mechanism of suppression in Rasdriven tumors. Elevated SPRY4 expression was also reported in response to NF1 deficiency in fibroblasts, and exogenous expression of SPRY2 induced senescence in NF1-deficient fibroblasts (17). DUSP and Sprouty genes are members of a 52-gene transcriptional profile that is specifically downstream of Raf/MEK/ERK signaling; these genes are expressed at high levels in tumor cell lines with an activating Raf mutation $\left({ }^{V 600 E} B R A F\right)$ but not tumor cell lines with receptor tyrosine kinase (RTK) activation (46). Consistent with our results in $N f 1^{f l / f l}$; Dhb-Cre neurofibromas, PD0325901 MEK inhibition reduces expression of these genes and blocks cell proliferation in tumor cell lines with an activating Raf mutation (40).

The PD0325901-induced reduction of neurofibroma volume in $39 / 49$ (80\%) of neurofibroma-bearing $N f 1^{f l / f l}$; Dhb-Cre mice represents the most dramatic result described to date for treatment of neurofibroma-bearing mice. In contrast, RAD001 did not decrease neurofibroma volume, and sorafenib, a multikinase inhibitor, decreased tumor volume in only 5 of 9 (56\%) mice (30). Imatinib, a tyrosine kinase inhibitor, showed an un-quantified effect on tumor burden in another neurofibroma mouse model (29). Plexiform neurofibromas have a slow growth rate and a complex nonspherical shape. Therefore, standard response criteria for malignant solid tumors have limited applicability. A more sensitive and reproducible method of response evaluation for human NF1 plexiform neurofibromas was developed, evaluating response and progression using volumetric MRI analysis. Response is defined as $20 \%$ or greater decrease in tumor volume compared with baseline, and progression is defined as $20 \%$ or greater increase in tumor volume compared with baseline (32). Plexiform neurofibroma shrinkage has not been observed in clinical trials, with the exception of up to $22 \%$ in response to pegintron (47) and rarely in response to imatinib (48). The method of volumetric MRI analysis used in our preclinical studies is identical to the method used in clinical trials, and we used identical response criteria.

Similar to our results, a previous study reported variable effects on MPNST cell survival in vitro with the MEK inhibitor from which PD0325901 was derived, PD184352 (CI-1040) (49). In contrast, the PD0325901 MEK inhibitor showed a robust, yet transient, in vivo effect on survival, possibly due to effects on tumor vasculature. We did not observe persistent apoptosis following PD0325901 treatment in vivo, in contrast with effects in vitro (49). Due to the multiplicity of Ras effectors and complexity of negative feedback regulation, therapeutic strategies against more aggressive Ras-related tumors are likely to include combinations of compounds that target multiple points in the Ras signaling network $(40,50-52)$. These studies support the investigation of combinatorial effects of PD0325901 with additional Ras pathway inhibitors in NF1 tumors.
Our results provide preclinical evidence implicating PD0325901 as a candidate NF1 therapeutic agent. This was unexpected, as the MEK inhibitor from which PD0325901 was derived failed to show efficacy in a GEM model of Nf1 JMML (53). Recently, PD0325901 was found to be effective in reversing myeloproliferative disease in an activated KRas mouse model (54) and in Nf1-driven JMML (33), likely due to the more persistent inhibition of MEK by PD0325901, a second-generation MEK inhibitor modified to improve efficacy for clinical cancer trials (55). Collectively, these studies suggest that Raf/MEK/ERK is a critical pathway in NF1.

Preliminary studies reported adverse effects after prolonged treatment of patients with advanced cancers with $10 \mathrm{mg}$ or more BID PD0325901 $(35,36,42)$. MPNST xenografts were only moderately sensitive to PD0325901 treatment at this dose, likely requiring combination therapies for MPNST treatment. However, the results of our experiments suggest that the lowest dose of $1.5 \mathrm{mg} / \mathrm{kg}$ in our preclinical neurofibroma mouse model, equivalent to $8 \mathrm{mg}$ BID in humans, is as effective in inhibiting tumor growth as the higher dose of $10 \mathrm{mg} / \mathrm{kg}$, supporting evaluation of new dosing schedules in clinical trials of PD0325901. Furthermore, the lower dose $(1.5 \mathrm{mg} / \mathrm{kg})$ appeared more effective in maintaining inhibition of MEK, with the higher dose activating the negative feedback response and elevating p-ERK to pretreatment levels. Fine-tuning the long-term maximal effective dose below the threshold of negative feedback may be relevant to monitoring PD0325901 in the clinic.

In summary, NF1 mutation causing neurofibromatosis results in hyperactive Ras, potentially activating numerous downstream signaling pathways. However, a successful targeted therapy in humans has not yet been developed. Combining mouse and human transcriptome data focused attention on increased expression of genes that suppress the Raf/MEK/ERK arm of Ras signaling. This transcriptional repression does not apparently compensate, as ERK phosphorylation was detected in neurofibromas and MPNST. Furthermore, inhibition of neurofibroma and MPNST growth with PD0325901 indicated dependence of both tumor types on sustained Raf/MEK/ ERK signaling. The results of our preclinical tests of PD0325901 in MPNST and neurofibroma support investigation of MEK inhibitors as candidate therapeutics in the treatment of Ras-related diseases, including NF1 and other "RASopathies" (56). In addition, NF1 mutations are frequently found in tumor types other than neurofibroma and MPNST, including glioblastoma (57), lung adenocarcinoma (58), and ovarian cancer (59). Thus, PD0325901 may be a potential molecular-targeted treatment for a wide variety of disorders.

\section{Methods}

Gene expression microarray. Mouse RNA isolation, hybridization and data normalization, mouse-human ortholog mapping and cross-species data integration are described in Supplemental Methods. Mouse microarray data can be accessed in the GEO MIAME-compliant public database (GSE41747).

In vitro MPNST cytotoxicity assay. MPNST cell lines (STS26T, ST8814, S462, S462TY, T265p21) were obtained and maintained as described (41). All MPNST cell lines were derived from NF1 patients except STS26T. MPNST cells were plated in quadruplicate for each dose of PD0325901 MEK inhibitor (gift of Pfizer Inc.). 24 hours after plating, cells were treated with vehicle alone (0.1\% DMSO) or inhibitor. Cell viability was quantified 96 hours after treatment by MTS assay as described (22).

Transient transfection of MPNST cells. Nucleofection and Western analyses are described in Supplemental Methods.

MPNST xenograft. The subcutaneous NF1-/- S462-TY human MPNST xenograft model has been previously described $(41,60)$. Administration 
and dose of PD0325901 were determined by previous animal studies (61). Daily oral gavage of vehicle $(0.5 \%[\mathrm{w} / \mathrm{v}]$ methylcellulose solution with $0.2 \%$ [v/v] polysorbate 80 [Tween 80 ] or $10 \mathrm{mg} / \mathrm{kg}$ PD0325901 in vehicle) was given for 28 days beginning when tumors reached $200 \mathrm{~mm}^{3} 3-4$ weeks after injection. We measured tumors and weighed mice twice weekly once tumors began enlarging. Tumor volume was calculated as follows: $L \times W 2$ $(\pi / 6)$, where $L$ is the longest diameter and $W$ is the width. Mice were treated until tumors reached $2500 \mathrm{~mm}^{3}$ or a maximum of 92 days. $100 \%$ of control tumors reached $2500 \mathrm{~mm}^{3}$. Two of 18 PD0325901-treated mice did not reach $2500 \mathrm{~mm}^{3}$; one showed complete and sustained remission at 150 days. Survival was analyzed by log-rank test using GraphPad Prism.

Neurofibroma MRI and tumor volume measurement. MRI and tumor volume measurement of $N f 1^{f / f l}$; $D$ hb-Cre mice was conducted as described (30). Mice were administered vehicle control $(0.5 \%[\mathrm{w} / \mathrm{v}]$ methylcellulose solution with $0.2 \%[\mathrm{v} / \mathrm{v}]$ polysorbate 80 [Tween 80$]$ or PD0325901 [1.5, 5.0, or $10 \mathrm{mg} / \mathrm{kg} / \mathrm{d}$ ]) by oral gavage for 60 days. Due to the high cost of performing serial MRI, littermate controls $(n=5)$ were allocated to the vehicle treatment group. In addition, historical controls $(n=20)$ were untreated or vehicle-treated control mice from the same genotype and strain background imaged over the 18 months preceding these experiments; these were included to increase statistical power (mixed effects model analysis).

MPNST statistical analysis. For MPNST, computing the mean and SEM based solely on surviving mice created downward bias, as mice with large tumors required sacrifice. We analyzed the longitudinally collected tumor volumes together across time points and conducted mixed effects analysis with autoregressive within-mouse dependent structure. Missing data from sacrificed mice with large tumors constituted a missing-at-completely-random (MACR) condition, under which the mixed effects analysis provided consistent estimates of mean tumor volume and SEM (62).

Immunohistochemistry. Paraffin sections were deparaffinized, hydrated and transferred to $0.1 \mathrm{M}$ citrate buffer ( $\mathrm{pH}$ 6.0) for antigen retrieval. Slides were boiled for 10 minutes in citrate buffer, cooled at room temperature for 30 minutes, then rinsed in water twice and in PBS 3 times. Sections were quenched with $3 \%$ hydrogen peroxide for 10 minutes, rinsed in PBS, and blocked in $10 \%$ normal goat serum with $0.3 \%$ Triton X-100. Sections were incubated overnight in primary antibody diluted in block; rabbit pERK (\#4370, 1:200, Cell Signaling; see Supplemental Methods), rabbit Ki67 (1:10,000; Novocastra), rat MECA 32 (1:5; Developmental Studies Hybridoma Bank), rabbit pS6Kinase (1:800; Cell Signaling), rabbit pAKT (1:50), and mouse CNPase (1:750; MAB226, Millipore). Sections were then incubated in appropriate biotinylated secondary antibodies; goat anti-rabbit or goat anti-rat (1:200; Vector). Some sections were counterstained with Harris hematoxylin. Numbers of $\mathrm{MECA}^{+}$blood vessels were counted in 10 $\times 20$ fields per MPNST $(n=5)$ and $5 \times 20$ fields per neurofibroma $(n=5)$ for each condition. Statistical comparisons between control and tumor samples were conducted using Student's $t$ test. All microscopic images were acquired with Openlab software suites on a Zeiss Axiovert 200.

For double labeling, paraffin sections were processed for antigen retrieval as above and incubated overnight in a cocktail of 2 of the following primary antibodies: rabbit p-ERK (1:200; 4370, Cell Signaling), mouse CNPase (1:750; MAB226, Millipore), rabbit Ki67 (1:1000; Novocastra), and rat MECA 32 (1:5; Developmental Studies Hybridoma Bank). Sections were then incubated with a cocktail of 2 appropriate secondary antibodies: Goat anti-rabbit Alexa Fluor 488 (1:800; Invitrogen), goat anti-mouse Alexa Fluor 594 (1:1200; Invitrogen), and goat anti-rat Alexa Fluor 594 (1:1200; Invitrogen). Sections were stained with DAPI $(1: 10,000)$ to visualize cell nuclei and mounted with Fluoromount G. Pictures were taken under a fluorescent microscope, and cells were counted using ImageJ software.

qRT-PCR. mRNAs were extracted from WT sciatic nerves, $N f^{1 / f l f} ; D h b$-Cre mouse neurofibromas, and PD0325901- or vehicle-treated $N f 1^{f / f f} ; D h b$-Cre mouse neurofibromas using the Mini-mRNA isolation kit (QIAGEN). The mRNA was reverse-transcribed using the Superscript Preamplification System (Gibco; Invitrogen). Superscript II reverse transcriptase was used in the reaction, as per the manufacturer's protocol. Duplicate samples lacking reverse transcriptase were conducted to control for genomic DNA contamination. Primers were designed and synthesized by Integrated DNA Technologies (IDT) for quantitative RT-PCR (qRT-PCR) of SPRY4 and DUSP6 (SPRY4F: 5'-TGTGAATCCCAGCTCAGTCATGGT-3'; SPRY4R: 5'-ATTCTCCACGTGGCTGGTCTTCAT-3'; DUSP6F: 5'-TGCCCAATCTGTTTGAGAATGCGG-3'; DUSP6R: 5'-CAATGCACCAGGACACCACAGTTT- $3^{\prime}$ ). Mouse tubulin primers (IDT) were included in each reaction as a positive control for CDNA. Triplicate reactions were performed in an ABI Prism 7500 as described (63). For statistical analyses, 3 mice were included for each treatment group. Relative gene expression was calibrated to tubulin expression. Fold change of SPRY4 or DUSP6 in untreated Nf1/f/fl; Dhh-Cre mouse neurofibromas compared with WT levels or in PD0325901-treated $N f 1^{f / f l} ; D h b$-Cre mouse neurofibromas compared with vehicle-treated $N f^{f / f / f}$; $D h h$-Cre mouse neurofibromas was calculated using the $\Delta \Delta \mathrm{C}_{\mathrm{T}}$ method.

Statistics. For gene expression microarray data analyses, ANOVA tests were used to identify transcripts differentially expressed; all tests were corrected for multiple testing effects by applying the Benjamini and Hochberg false discovery rate correction. Significance was accepted at $P<0.05$ for all biological experiments. MPNST survival analyses used Kaplan Meier analysis followed by a Gehan-Breslow-Wilcox log-rank test. Neurofibroma growth was modeled by Mixed Effects Model Analysis; we generated $P$ values with a random effects model analysis on log transformed tumor volume data using the SAS mixed procedure. Proliferation, cell death, and blood vessel quantification used 2-tailed Student's $t$ test.

Study approval. Human paraffin-embedded tissues were collected under Cincinnati Children's Hospital Medical Center (CCHMC) IRB approval. Informed consent was not required, as we used archival samples from a tissue bank that remained anonymous. The CCHMC Animal Use and Care Committee approved all animal use.

\section{Acknowledgments}

We thank Jan Manent for processing human nerve samples, Kevin Shannon (UCSF) for numerous helpful discussions and providing the mutant MEK L115P construct, Pfizer for providing PD0325901 for preclinical testing, and Mila McCurrach for organization of the CTF Preclinical Consortium. We thank Margaret Collins (CCHMC) for providing human tissue samples. We gratefully acknowledge support from the DAMD Program on Neurofibromatosis for the NF1 Microarray Consortium (DODW81XWH- 09-1-0135 and W81XWH-04-1-0273 to N. Ratner) and the Children's Tumor Foundation for support to the NF1 Preclinical Consortium (to T.P. Cripe and N. Ratner), and a Bench to Bedside Award for MRI Analyses (NIH-P50-NS05753). W.J. Jessen was supported in part by an ARRA supplement to NIH grant R01-NS28840.

Received for publication January 25, 2012, and accepted in revised form October 23, 2012.

Address correspondence to: Nancy Ratner, Children's Hospital Medical Center, Division of Experimental Hematology and Cancer Biology, 3333 Burnet Ave., M.L.C. 7013, Cincinnati, Ohio 45229, USA. Phone: 513.636.9469; Fax: 513.636.3549; E-mail: Nancy.Ratner@cchmc.org.

Walter J. Jessen's present address is: Biomarker Center of Excellence, Covance, Greenfield, Indiana, USA. 
1. Theos A, Korf BR. Pathophysiology of neurofibromatosis type 1. Ann Intern Med. 2006;144(11):842-849.

2. De Raedt T, et al. Somatic loss of wild type NF1 allele in neurofibromas: comparison of NF1 microdeletion and non-microdeletion patients. Genes Chromosomes Cancer. 2006;45(10):893-904.

3. Carli $M$, et al. Pediatric malignant peripheral nerve sheath tumor: the Italian and German soft tissue sarcoma cooperative group. J Clin Oncol. 2005; 23(33):8422-8430

4. Evans DG, Baser ME, McGaughran J, Sharif S, Howard E, Moran A. Malignant peripheral nerve sheath tumours in neurofibromatosis $1 . \mathrm{J} \mathrm{Med}$ Genet. 2002;39(5):311-314.

5. Friedrich RE, Hartmann M, Mautner VF. Malignant peripheral nerve sheath tumors (MPNST) in NF1-affected children. Anticancer Res. 2007 27(4A):1957-1960.

6. McCaughan JA, Holloway SM, Davidson R, Lam WW. Further evidence of the increased risk for malignant peripheral nerve sheath tumour from a Scottish cohort of patients with neurofibromatosis type 1. J Med Genet. 2007;44(7):463-466.

7. Huson SM, et al. Back to the future: proceedings from the 2010 NF Conference. Am J Med Genet A. 2011 $155 \mathrm{~A}(2): 307-321$

8. Carroll SL, Ratner N. How does the Schwann cell lineage form tumors in NF1? Glia. 2008; 56(14):1590-1605

9. McCormick F. Ras signaling and NF1. Curr Opin Genet Dev. 1995;5(1):51-55.

10. Le LQ, Parada LF. Tumor microenvironment and neurofibromatosis type I: connecting the GAPs. Oncogene. 2007;26(32):4609-4616.

11. Sherman LS, Atit R, Rosenbaum T, Cox AD, Ratner N. Single cell Ras-GTP analysis reveals altered Ras activity in a subpopulation of neurofibroma Schwann cells but not fibroblasts. J Biol Chem. 2000; 275(39):30740-30745.

12. Basu TN, Gutmann DH, Fletcher JA, Glover TW, Collins FS, Downward J. Aberrant regulation of ras proteins in malignant tumour cells from type 1 neurofibromatosis patients. Nature. 1992; 356(6371):663-664.

13. DeClue JE, et al. Abnormal regulation of mammalian $\mathrm{p} 21^{\text {ras }}$ contributes to malignant tumor growth in von Recklinghausen (Type 1) neurofibromatosis. Cell. 1992;69(2):265-273.

14. Downward J. Targeting RAS signalling pathways in cancer therapy. Nat Rev Cancer. 2003;3(1):11-22.

15. Collado $M$, et al. Tumour biology: senescence in premalignant tumours. Nature. 2005; 436(7051):642.

16. Narita M, Lowe SW. Senescence comes of age. Nat Med. 2005;11(9):920-922.

17. Courtois-Cox $S$, et al. A negative feedback signaling network underlies oncogene-induced senescence. Cancer Cell. 2006;10(6):459-472.

18. Cox AD, Der CJ. Ras history: the saga continues. Small Gtpases. 2010;1(1):2-27.

19. Katz D, Lazar A, Lev D. Malignant peripheral nerve sheath tumour (MPNST): the clinical implications of cellular signalling pathways. Expert Rev Mol Med. 2009;11:e30.

20. Weiss B, Bollag G, Shannon K. Hyperactive Ras as a therapeutic target in neurofibromatosis type $1 . A m$ JMed Genet. 1999;89(1):14-22.

21. Bodempudi $V$, et al. Ral overactivation in malignant peripheral nerve sheath tumors. Mol Cell Biol. 2009; 29(14):3964-3974.

22. Johansson $\mathrm{G}$, et al. Effective in vivo targeting of the mammalian target of rapamycin pathway in malignant peripheral nerve sheath tumors. Mol Cancer Ther. 2008;7(5):1237-1245.

23. Johannessen CM, Reczek EE, James MF, Brems H,
Legius E, Cichowski K. The NF1 tumor suppressor critically regulates TSC2 and mTOR. Proc Natl Acad Sci U S A. 2005;102(24):8573-8578.

24. Zhu Y, Ghosh P, Charnay P, Burns DK, Parada LF. Neurofibromas in NF1: Schwann cell origin and role of tumor environment. Science. 2002; 296(5569):920-922.

25. Wu J, et al. Plexiform and dermal neurofibromas and pigmentation are caused by Nf1 loss in desert hedgehog-expressing cells. Cancer Cell. 2008; 13(2):105-116

26. Zheng $\mathrm{H}$, et al. Induction of abnormal proliferation by nonmyelinating schwann cells triggers neurofibroma formation. Cancer Cell. 2008;13(2):117-128.

27. Joseph NM, et al. The loss of Nf1 transiently promotes self-renewal but not tumorigenesis by neural crest stem cells. Cancer Cell. 2008;13(2):129-140.

28. Gitler AD, et al. Nf1 has an essential role in endothelial cells. Nat Genet. 2003;33(1):75-79.

29. Yang FC, et al. Nf1-dependent tumors require a microenvironment containing $\mathrm{Nf}^{+/-}$and c-kitdependent bone marrow. Cell. 2008;135(3):437-448.

30. Wu J, et al. Preclincial testing of Sorafenib and RAD001 in the Nf(flox/flox);DhhCre mouse model of plexiform neurofibroma using magnetic resonance imaging. Pediatr Blood Cancer. 2012; 58(2):173-180.

31. Solomon J, Warren K, Dombi E, Patronas N, Widemann B. Automated detection and volume measurement of plexiform neurofibromas in neurofibromatosis 1 using magnetic resonance imaging. Comput Med Imaging Graph. 2004;28(5):257-265.

32. Dombi E, et al. NF1 plexiform neurofibroma growth rate by volumetric MRI: relationship to age and body weight. Neurology. 2007;68(9):643-647.

33. Chang T, et al. Sustained MEK inhibition abrogates myeloproliferative disease in $N f 1$ mutant mice. J Clin Invest. 2013;123(1):335-339.

34. Miller SJ, et al. Integrative genomic analyses of neurofibromatosis tumours identify SOX9 as a biomarker and survival gene. EMBO Mol Med. 2009; 1(4):236-248.

35. Haura EB, et al. A phase II study of PD-0325901, an oral MEK inhibitor, in previously treated patients with advanced non-small cell lung cancer. Clin Cancer Res. 2010;16(8):2450-2457

36. LoRusso PM, et al. Phase I pharmacokinetic and pharmacodynamic study of the oral MAPK/ERK kinase inhibitor PD-0325901 in patients with advanced cancers. Clin Cancer Res. 2010;16(6):1924-1937.

37. Bain J, et al. The selectivity of protein kinase inhibitors: a further update. Biochem J. 2007; 408(3):297-315.

38. Sebolt-Leopold JS, Herrera R. Targeting the mitogen-activated protein kinase cascade to treat cancer. Nat Rev Cancer. 2004;4(12):937-947.

39. Mahller YY, Rangwala F, Ratner N, Cripe TP. Malignant peripheral nerve sheath tumors with high and low Ras-GTP are permissive for oncolytic herpes simplex virus mutants. Pediatr Blood Cancer. 2006; 46(7):745-754.

40. Solit DB, et al. BRAF mutation predicts sensitivity to MEK inhibition. Nature. 2006;439(7074):358-362.

41. Patel AV, et al. Ras-driven transcriptome analysis identifies aurora kinase a as a potential malignant peripheral nerve sheath tumor therapeutic target. Clin Cancer Res. 2012;18(18):5020-5030.

42. Boasberg PD, Redfern CH, Daniels GA, Bodkin D, Garrett CR, Ricart AD. Pilot study of PD-0325901 in previously treated patients with advanced melanoma, breast cancer, and colon cancer. Cancer Chemother Pharmacol. 2011;68(2):547-552.

43. Wu J, et al. Preclincial testing of sorafenib and RAD001 in the Nf(flox/flox);DhhCre mouse model of plexiform neurofibroma using magnetic resonance maging. Pediatr Blood Cancer. 2012;58(2):173-180.

44. Ciuffreda L, et al. Growth-inhibitory and antiangiogenic activity of the MEK inhibitor PD0325901 in malignant melanoma with or without BRAF mutations. Neoplasia. 2009;11(8):720-731.

45. Sweet-Cordero A, et al. An oncogenic KRAS2 expression signature identified by cross-species geneexpression analysis. Nat Genet. 2005;37(1):48-55.

46. Pratilas CA, et al. (V600E)BRAF is associated with disabled feedback inhibition of RAF-MEK signaling and elevated transcriptional output of the pathway. Proc Natl Acad Sci U S A. 2009;106(11):4519-4524.

47. Jakacki RI, et al. Phase I trial of pegylated interferon-alpha- $2 b$ in young patients with plexiform neurofibromas. Neurology. 2011;18(3):265-272.

48. Robertson KA, et al. Phase II pilot study of imatinib mesylate in neurofibromatosis (NF1) patients with plexiform neurofibromas. J Clin Oncol. 2011; 29(suppl; abstract 10030).

49. Mattingly RR, et al. The mitogen-activated protein kinase/extracellular signal-regulated kinase kinase inhibitor PD184352 (CI-1040) selectively induces apoptosis in malignant schwannoma cell lines. J Pharmacol Exp Ther. 2006;316(1):456-465.

50. Gysin S, Salt M, Young A, McCormick F. Therapeutic strategies for targeting ras proteins. Genes Cancer. 2011;2(3):359-372.

51. Sos ML, et al. Identifying genotype-dependent efficacy of single and combined PI3K- and MAPK-pathway inhibition in cancer. Proc Natl Acad Sci U S A. 2009;106(43):18351-18356

52. Gioeli D, et al. Compensatory pathways induced by MEK inhibition are effective drug targets for combination therapy against castration-resistant prostate cancer. Mol Cancer Ther. 2011;10(9):1581-1590.

53. Lauchle JO, et al. Response and resistance to MEK inhibition in leukaemias initiated by hyperactive Ras. Nature. 2009;461(7262):411-414.

54. Lyubynska $\mathrm{N}$, et al. A MEK inhibitor abrogates myeloproliferative disease in Kras mutant mice. Sci Transl Med. 2011;3(76):76ra27.

55. Sebolt-Leopold JS, English JM. Mechanisms of drug inhibition of signalling molecules. Nature. 2006; 441(7092):457-462

56. Rauen KA, et al. Costello and cardio-facio-cutaneous syndromes: Moving toward clinical trials in RASopathies. Am J Med Genet C Semin Med Genet. 2011;157(2):136-146.

57. Masica DL, Karchin R. Correlation of somatic mutation and expression identifies genes important in human glioblastoma progression and survival. Cancer Res. 2011;71(13):4550-4561.

58. Ding L, et al. Somatic mutations affect key pathways in lung adenocarcinoma. Nature. 2008; 455(7216):1069-1075

59. Cancer Genome Atlas Research Network. Integrated genomic analyses of ovarian carcinoma. Nature. 2011;474(7353):609-615.

60. Mahller YY, et al. Tissue inhibitor of metalloproteinase-3 via oncolytic herpesvirus inhibits tumor growth and vascular progenitors. Cancer Res. 2008; 68(4):1170-1179

61. Brown AP, Carlson TC, Loi CM, Graziano MJ. Pharmacodynamic and toxicokinetic evaluation of the novel MEK inhibitor, PD0325901, in the rat following oral and intravenous administration. Cancer Chemother Pharmacol. 2007;59(5):671-679.

62. Liu C, Cripe TP, Kim MO. Statistical issues in longitudinal data analysis for treatment efficacy studies in the biomedical sciences. Mol Ther. 2010; 18(9):1724-1730.

63. Miller SJ, et al. Large-scale molecular comparison of human schwann cells to malignant peripheral nerve sheath tumor cell lines and tissues. Cancer Res. 2006;66(5):2584-2591. 